\title{
In Vitro Analysis and In Vivo Tumor Targeting of a Humanized, Grafted Nanobody in Mice Using Pinhole SPECT/Micro-CT
}

Ilse Vaneycken ${ }^{1,2}$, Jochen Govaert ${ }^{3,4}$, Cécile Vincke ${ }^{3,4}$, Vicky Caveliers ${ }^{1,2}$, Tony Lahoutte ${ }^{1,2}$, Patrick De Baetselier ${ }^{3,4}$, Geert Raes $^{3,4}$, Axel Bossuyt ${ }^{1,2}$, Serge Muyldermans ${ }^{3,4}$, and Nick Devoogdt ${ }^{1,3,4}$

${ }^{1}$ Laboratory for In Vivo Cellular and Molecular Imaging, Vrije Universiteit Brussel (VUB), Brussels, Belgium; ${ }^{2}$ Nuclear Medicine Department, UZ Brussel, Brussels, Belgium; ${ }^{3}$ Department of Molecular and Cellular Interactions, Vlaams Instituut voor Biotechnologie (VIB), Brussels, Belgium; and ${ }^{4}$ Department of Cellular and Molecular Immunology, Vrije Universiteit Brussel (VUB), Brussels, Belgium

Nanobodies are a novel type of immunoglobulinlike, antigenbinding protein with beneficial pharmacologic and pharmacokinetic properties that are ideally suited to targeting cellular antigens for molecular imaging or therapeutic purposes. However, because of their camelid, nonhuman origin, the possible immunogenicity of Nanobodies when used in the clinic is a concern. Here we present a new strategy to quickly generate humanized Nanobodies for molecular imaging purposes. Methods: We genetically grafted the antigen-binding loops of NbCEA5, a Nanobody with specificity for the colon carcinoma marker carcinoembryonic antigen (CEA), onto the framework of a humanized Nanobody scaffold. This scaffold has been previously characterized in our laboratory as a stable Nanobody that can serve as a universal loop acceptor for antigen-binding loops from donor Nanobodies and has been additionally mutated at about 10 crucial surface-exposed sites to resemble the sequence of human variable immunoglobulin domains. The 3 recombinant Nanobodies (NbCEA5, humanized scaffold, and humanized CEA5 graft) were produced in bacteria and purified. Unlabeled and ${ }^{99 \mathrm{~m} T c}$-labeled Nanobodies were biochemically characterized in vitro and tested as probes for SPECT/CT of xenografted tumors. Results: The success of loop-grafting was confirmed by comparing these Nanobodies for their capacity to recognize soluble CEA protein in enzyme-linked immunosorbent assay and by surface plasmon resonance and to bind to CEA-positive LS174T colon carcinoma cells and CEAtransfected but not untransfected Chinese hamster ovary cells in flow cytometry. Specificity of binding was confirmed by competition studies. All Nanobodies were heat-stable, could be efficiently labeled with $99 \mathrm{mTc}$, and recognized both soluble and membrane-bound CEA protein in binding studies. Finally, biodistribution experiments were performed with intravenously injected $99 \mathrm{~m}$ Tc-labeled Nanobodies in LS174T tumor-bearing mice using pinhole SPECT/micro-CT. These in vivo experiments revealed specificity of tumor targeting and rapid renal clearance for all Nanobodies, with low signals in all organs besides the kidneys. Conclusion: This study shows the potency of antigen-

Received Aug. 26, 2009; revision accepted Mar. 4, 2010.

For correspondence or reprints contact: Ilse Vaneycken, Laboratory for In Vivo Cellular and Molecular Imaging, Vrije Universiteit Brussel, Laarbeeklaan 103, 1090 Brussels, Belgium.

E-mail: ilse.vaneycken@gmail.com

COPYRIGHT $\odot 2010$ by the Society of Nuclear Medicine, Inc. binding loop-grafting to efficiently generate humanized Nanobodies that retain their targeting capacities for noninvasive in vivo imaging of tumors.

Key Words: 99mTc-Nanobody; humanization; CEA; SPECT/CT; biodistribution; oncology; animal imaging

J Nucl Med 2010; 51:1099-1106

DOI: 10.2967/jnumed.109.069823

$\mathbf{N}$ anobodies (trademarked by Ablynx) are small antigenbinding single-domain proteins derived from the variable fragment of unique heavy-chain-only antibodies that are naturally present in sera of Camelidae (1). Besides their small size $(15 \mathrm{kDa})$ and a rapid blood clearance, Nanobodies offer many advantages for use as targeted tracers, including high affinity and specificity for their cognate antigen, high solubility and stability, facile production, and radiolabeling (2-6). Indeed, our previous work with Nanobodies targeting carcinoembryonic antigen (CEA) (7) or epidermal growth factor receptor $(8,9)$ demonstrated that Nanobodies bind tumor antigens rapidly and specifically in vivo, whereas untargeted Nanobody is readily cleared from the blood, obtaining high tumor-to-background ratios early $(1 \mathrm{~h})$ after tracer injection. However, the nonhuman origin of Nanobodies might elicit a neutralizing anti-Nanobody immune response in humans, similar to the human antiglobulin antibody response. Although no experimental data exist to substantiate such anti-Nanobody human antiglobulin antibody response, it could prevent the Nanobody from binding to its target and may cause allergiclike symptoms when administered repeatedly (10). Nanobodies most likely have low immunogenicity because of their rapid blood clearance and high sequence identity to human variable domain of the heavy chain $\left(\mathrm{V}_{\mathrm{H}} \mathrm{s}\right)$. Nevertheless, a Nanobody differs from a human $\mathrm{V}_{\mathrm{H}}$ in about 10 amino acids spread over its surface to ensure maximal solubility and stability in the absence of 
a human variable domain of the light chain domain (11-14). Therefore, for clinical applications, further humanization of the Nanobodies must be considered.

Ideally, a humanization of a Nanobody should result in a derivative that is nonimmunogenic, with complete retention of the antigen-binding properties of the original molecule. To accomplish this, the structure of the Nanobody antigen-binding site has to be maintained in the humanized version. This preservation of the Nanobody antigen-binding site can be potentially achieved by transplanting the binding site of the nonhuman Nanobody onto a human framework, as we have shown before (15), or by resurfacing the framework of the Nanobody of interest.

In this study, the antigen-binding loops of Nanobody NbCEA5 (i.e., a Nanobody with specificity and strong binding to CEA - a biomarker for gastrointestinal, breast, lung, and ovarian carcinomas (16)) were genetically grafted onto the framework of a humanized Nanobody scaffold. This scaffold has been mutated at 13 crucial surfaceexposed sites to maximally resemble human $\mathrm{V}_{\mathrm{H}}$ sequences and previously characterized as a highly stable, humanized Nanobody that serves as a universal loop-acceptor to graft antigen-binding loops from donor Nanobodies with retention of the antigen-specificity of the loop-donor Nanobody (15). The aim of this report was to compare in vitro characteristics, in vivo tumor uptake, and biodistribution using pinhole SPECT and micro-CT imaging of 3 Nanobodies: NbCEA5, the humanized Nanobody scaffold, and the humanized CEA5 graft.

\section{MATERIALS AND METHODS}

\section{Generation and Purification of NbCEA5, Humanized Scaffold, and Humanized CEA5 Graft}

NbCEA5 (previously referred to as cAb-CEA5) was obtained after immunizing a dromedary with CEA protein, cloning the Nanobodies from its lymphocytes, and selecting the Nanobodies that bind to CEA protein by phage display (4). The humanized scaffold (previously referred to as h-NbBcII10 $0_{\text {FGLA }}$ ) was generated by mutating 13 residues in NbBcII10 (a Nanobody targeting a bacterial enzyme) to resemble more closely human $V_{H}$ sequences (15). The humanized CEA5 graft was generated by polymerase chain reaction-based mutagenesis, essentially as described in Vincke et al. (15) and Saerens et al. (17). Briefly, the sequence of each antigen-binding loop (also called complementdetermining region [CDR]) from NbCEA5 was encompassed by 2 primers, at its $3^{\prime}$ ends encoding for the framework regions (FRs) of the humanized scaffold. Thus, the indicated chimeric fragments were amplified using humanized scaffold DNA as a template and the following primer pairs: 5'-GCC CAG CCG GCC ATG GCC CAG GTG CAG CTG GTG-3' and 5'-TCC TGT GCA GCC TCT GGA GAC ACC TAC GGT AGT TAC-3' (FR1 $1_{\text {humanized scaffold }}{ }^{-}$ CDR $1_{\text {NbCEA }}$ ); 5'-TAC GGT AGT TAC TGG ATG GGC TGG TTC CGC CAG-3' and 5'-GCG GTC GCG GCT ATC AAT AGG GGT GGT GGC-3' ${ }^{\prime}$ (CDR $1_{\text {NbCEA }}-\mathrm{FR} 2_{\text {humanized }}$ scaffold ${ }^{-}$ CDR2 ${ }_{\text {NbCEA5 }}$ ); 5'-AGG GGT GGT GGC TAT ACA GTC TAC GCC GAC-3' and 5'-TAT TAC TGT GCG GCG AGC GGG GTA CTA GGT GGT TTA CAT GAG GAC-3' (CDR2 $_{\text {NbCEA }^{-}}$ FR3 $3_{\text {humanized scaffold }}-\mathrm{CDR} 3_{\text {NbCEA5 }}$ ); and $5^{\prime}$-GGT GGT TTA CAT
GAG GAC TGG TTT AAC TAC TGG GGC CAG GGG ACC CTG GTC ACC GTC-3' and 5' - GTA AAA CGA CGG CCA GT3' (CDR3 $\left.3_{\text {NbCEA }}-\mathrm{FR} 4_{\text {humanized scaffold }}\right)$. The resulting DNA fragments were linked using splicing by overlap extension polymerase chain reaction to generate the humanized CEA5 graft DNA fragment, digested with enzymes NcoI and BstEII (Fermentas) and cloned in plasmid pHEN6c (18). Also, Nanobody genes coding for NbCEA5 and the humanized scaffold were cloned in this bacterial expression vector pHEN6c. The expression products contained a carboxy C-terminal hexahistidine $\left(\mathrm{His}_{6}\right)$ tag. A variant of NbCEA5 was also generated with a combination of the $\mathrm{His}_{6}$ and a C-terminal Myc tag. All Nanobody proteins were purified from Escherichia coli periplasmic extracts using immobilized metal affinity chromatography on Ni-NTA resin (Sigma-Aldrich), followed by size-exclusion chromatography on Superdex $75 \mathrm{HR}$ 10/30 (Pharmacia) in phosphate-buffered saline (PBS), pH 7.4, as described previously (17). An aliquot was taken after each purification step, separated by sodium dodecylsulfate-polyacrylamide gel electrophoresis under reducing and denaturing conditions and stained with Coomassie blue.

\section{In Vitro Characterization of NbCEA5, Humanized Scaffold, and Humanized CEA5 Graft}

The binding properties of NbCEA5, humanized scaffold, and humanized CEA5 graft were measured by surface plasmon resonance (SPR) on the BIAcore 3000 instrument (GE Healthcare) (4). Briefly, purified CEA protein (Fitzgerald Industries) was coupled onto a CM5 chip (GE Healthcare) via 1-ethyl-3-[3-dimethylaminopropyl]carbodiimide and hydrochloride- $N$-hydroxysuccinimide chemistry. The affinity of the Nanobodies for the CEA target was measured via kinetic titration using Nanobody concentrations ranging from 2 to $500 \mathrm{nM}$. Association and dissociation rate constants were obtained using BIAevaluation software (version 4.1; BIAcore) and used to calculate the affinity (equilibrium dissociation constant).

As described before (15), the melting temperature of the Nanobody was determined by following the circular dichroism signal of the unfolding protein induced by an increase in temperature on a $\mathbf{J} 715$ spectropolarimeter (Jasco). Melting curves were recorded from $35^{\circ} \mathrm{C}$ to $95^{\circ} \mathrm{C}$, with a temperature gradient of $1^{\circ} \mathrm{C} / \mathrm{min}$ at a fixed wavelength of $205 \mathrm{~nm}$. A protein concentration between 0.1 and $0.2 \mathrm{mg} / \mathrm{mL}$ in $50 \mathrm{mM}$ phosphate buffer, $\mathrm{pH} 7.0$, was used. A cuvette with a $0.1-\mathrm{cm}$ cell path length was used, and 1 data point was acquired every $20 \mathrm{~s}$, with a 1-s integration time and 2-nm bandwidth. Data analysis was performed as described previously (15).

Binding of the 3 Nanobodies to Chinese hamster ovary $(\mathrm{CHO})$ cells, CEA-transfected CHO, and CEA-expressing LS174T cells was investigated by flow cytometry. Nanobody binding was detected by adding sequentially an anti-His 6 antibody (AbD Serotec) and phycoerythrine-labeled antimouse IgG antibody (Becton Dickinson). One microgram of Nanobody was used per million cells. Binding of $100 \mu \mathrm{L}$ of Nanobody $(1 \mu \mathrm{g} / \mathrm{mL})$ to immobilized CEA was assessed by enzyme-linked immunosorbent assay (ELISA) and detected with anti-His 6 antibody (AbD Serotec) and color conversion of substrate by alkaline phosphatase conjugated to anti-mouse IgG antibody (Sigma-Aldrich).

The specificity of the Nanobodies was investigated by competition studies in ELISA and flow cytometry. To this end, we used 1 $\mu \mathrm{g}$ of NbCEA5-Myc Nanobody per milliliter for ELISA or $1 \mu \mathrm{g}$ of NbCEA5-Myc Nanobody per million cells for flow cytometry. 
NbCEA5-Myc Nanobody is identical to NbCEA5 but with an additional C-terminal Myc tag. Binding of the NbCEA5-Myc Nanobody to CEA, with or without a 10 -fold excess of nonMyc-tagged NbCEA5, humanized scaffold, or humanized CEA5 graft, was visualized using an anti-Myc antibody (AbD Serotec) in both assays.

\section{Cell Culture and Animal Model}

The human colon adenocarcinoma cell line LS174T was obtained from American Type Culture Collection and expresses large amounts of membrane-bound CEA. CHO-CEA cells have been described previously and were kindly provided by Dr. Motomu Kuroki (19). LS174T cells were cultured in Eagle's minimal essential medium (Gibco BRL) supplemented with 10\% heat-inactivated fetal calf serum, $2 \mathrm{mM}$ L-glutamine, $1 \mathrm{mM}$ sodium pyruvate, $1 \mathrm{mM}$ nonessential amino acids, 100 units of penicillin per milliliter, and $0.1 \mathrm{mg}$ of streptomycin per milliliter. $\mathrm{CHO}$ and $\mathrm{CHO}-\mathrm{CEA}$ cells were cultured in RPMI medium (Gibco BRL) supplemented with $10 \%$ heat-inactivated fetal calf serum, 2 $\mathrm{mM}$ L-glutamine, 100 units of penicillin per milliliter, and $0.1 \mathrm{mg}$ of streptomycin per milliliter. All cells were detached using trypsin-ethylenediaminetetraacetic acid.

The LS174T cells $\left(1 \times 10^{6}\right)$ in $200 \mu \mathrm{L}$ of PBS were subcutaneously injected into the right hind leg of male nude $n u / n u$ athymic mice (age, $6 \mathrm{wk}$ ) under the control of $2.5 \%$ isoflurane (Abbott). Tumors were allowed to grow for 2 wk to reach a diameter of approximately $0.5-1 \mathrm{~cm}$. The animal study protocol was approved by the local ethical committee for animal research.

\section{Nanobody Labeling and In Vitro Characterization of 99mTc-Labeled Nanobodies}

The 3 Nanobodies were labeled with ${ }^{99 \mathrm{~m}} \mathrm{Tc}$ at their $\mathrm{His}_{6}$ tail, as described previously $(8,9)$. Briefly, $\left[{ }^{99} \mathrm{~m} \mathrm{Tc}\left(\mathrm{H}_{2} \mathrm{O}\right)_{3}(\mathrm{CO})_{3}\right]^{+}$was synthesized by adding $1 \mathrm{~mL}$ of fresh ${ }^{99} \mathrm{mcO}_{4}{ }^{-}$eluate $(0.74-3.7$ $\mathrm{GBq}$ ) from a ${ }^{99} \mathrm{Mo}-{ }^{99 \mathrm{~m}} \mathrm{Tc}$ generator (Drytec; GE Healthcare) to an Isolink kit (Mallinckrodt Medical BV); the mixture was boiled for $20 \mathrm{~min}$. After neutralization with $1 \mathrm{~N} \mathrm{HCl},\left[{ }^{99 \mathrm{~m}} \mathrm{Tc}\left(\mathrm{H}_{2} \mathrm{O}\right)_{3}(\mathrm{CO})_{3}\right]^{+}$ was added to a $1 \mathrm{mg} / \mathrm{mL}$ Nanobody solution and was incubated for $90 \mathrm{~min}$ at $52^{\circ} \mathrm{C}$. After this labeling step, the ${ }^{99 \mathrm{~m}} \mathrm{Tc}-$ Nanobody solution was purified on a NAP-5 column (GE Healthcare) preequilibrated with $\mathrm{PBS}$ to remove unbound $\left[{ }^{99 \mathrm{~m}} \mathrm{Tc}\left(\mathrm{H}_{2} 0\right)_{3}(\mathrm{CO})_{3}\right]^{+}$and passed through a $0.22-\mu \mathrm{m}$ Millipore filter to eliminate possible aggregates. The labeling efficiency was determined both directly after labeling and after purifications by instant thin-layer chromatography with $100 \%$ acetone as the mobile phase.

To confirm the antigen specificity of the Nanobody after labeling, adherent $\mathrm{CHO}$ (negative control) or CEA-transfected $\mathrm{CHO}$ cells were fixed in $4 \%$ formaldehyde and incubated with $0.15 \mu \mathrm{g}(0.37-0.55 \mathrm{MBq})$ of labeled Nanobody for $1 \mathrm{~h}$ at room temperature. Bound Nanobody was washed with $10 \%$ fetal calf serum in PBS and then eluted with 0.1 M Tris-glycine, $\mathrm{pH}$ 2.7. Cell-associated radioactivity was measured in an automated $\gamma$-counter (Cobra II Inspector 5003; Canberra-Packard).

\section{Pinhole SPECT/micro-CT Imaging Procedure}

LS174T xenografts (6 mice per Nanobody) were intravenously injected with $45-155 \mathrm{MBq}(2.5 \mu \mathrm{g})$ of either $99 \mathrm{~m}$ Tc-NbCEA5, ${ }^{99 \mathrm{~m}}$ Tc-humanized scaffold, or ${ }^{99 \mathrm{~m}} \mathrm{Tc}$-humanized CEA5 graft. Mice were anesthetized with an $18.75 \mathrm{mg} / \mathrm{kg}$ mixture of ketamine hydrochloride (Ketamine 1000; CEVA) and $0.5 \mathrm{mg}$ of medetomi- dine hydrochloride per kilogram (Domitor; Pfizer) 10-15 min before pinhole SPECT acquisition.

Micro-CT imaging was followed by pinhole SPECT on separate systems. Micro-CT was performed using a dual-source CT scanner (Skyscan 1178; Skyscan) with $60 \mathrm{kV}$ and $615 \mathrm{~mA}$ at a resolution of $83 \mu \mathrm{m}$. The total body scan time was $2 \mathrm{~min}$. Images were reconstructed using filtered backprojection (NRecon; Skyscan). Total body pinhole SPECT was performed once at $60 \mathrm{~min}$ after injection using a dual-head $\gamma$-camera (e.cam ${ }^{180}$; Siemens Medical Solutions), mounted with 2 multipinhole collimators (three $1.5-\mathrm{mm}$ pinholes in each collimator, 200-mm focal length, and $80-\mathrm{mm}$ radius of rotation). Images were acquired over $360^{\circ}$ in 64 projections of $10 \mathrm{~s}$ into $128 \times 128$ matrices, resulting in a total imaging time of $14 \mathrm{~min}$. The SPECT images were reconstructed using an iterative reconstruction algorithm (ordered-subset expectation maximization) modified for the 3-pinhole geometry and automatically reoriented for fusion with CT images based on six ${ }^{57}$ Co landmarks (20).

\section{Image Analysis}

Images were viewed and quantified using AMIDE: a Medical Image Data Examiner software (21). Ellipsoid regions of interest based on the CT images were drawn on muscle and around the total body. Because of insufficient contrast on the CT images, SPECT images were used to draw a region of interest around the tumor and kidney and on the liver and lungs. For delineation of the tumor, a threshold of $50 \%$ or more of the maximum pixel value on the SPECT images was chosen. The counts measured at the injection site were subtracted from the total body counts. Uptake was calculated as the counts in the tissue divided by the injected activity counts and normalized for the region of interest (\%IA/ $\left.\mathrm{cm}^{3}\right)$.

\section{Blood Clearance of $99 \mathrm{~m}$ Tc-Labeled Nanobodies}

In a separate group of naïve athymic nude mice ( 2 mice per Nanobody), blood samples were collected using a microcapillary at $1,5,10,20,40,60,90$, and $120 \mathrm{~min}$ after injection of ${ }^{99 \mathrm{~m}} \mathrm{Tc}-$ labeled Nanobodies to obtain a time-activity curve. Data are presented as percentage of injected activity (\%IA) per total blood volume. Total blood volume was calculated as $7 \%$ of the total body weight.

\section{Statistical Analyses}

All statistical analyses were performed using the unpaired, 2-tailed $t$ test.

\section{RESULTS}

\section{In Vitro Characterization of NbCEA5, Humanized} Scaffold, and Humanized CEA5 Graft

The 3 antigen-binding loops (CDRs) of the CEA-binding Nanobody NbCEA5 were genetically grafted onto the FRs of a humanized Nanobody scaffold. Of the 3 Nanobodies that were used in this study, the CDRs of NbCEA5 and humanized CEA5 graft are identical, whereas the humanized scaffold and humanized CEA5 graft share the same FRs (Fig. 1).

The recombinant Nanobodies NbCEA5, humanized scaffold, and humanized CEA5 graft were purified from Escherichia coli periplasmic extracts by immobilized metal affinity chromatography, followed by size-exclusion chromatography 
FIGURE 1. Amino acid sequences of $\mathrm{His}_{6}$-tagged Nanobody NbCEA5, humanized CEA5 graft, and humanized scaffold. FRs 1-4 and antigen-binding loops (CDRs 1-3) are indicated. Humanizing mutations in FRs are in bold italic; CEA-targeting CDRs are in bold.

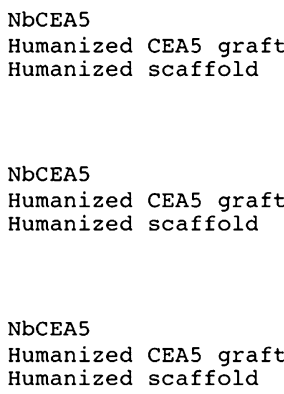

$\frac{\text { FR1 }}{\text { OVQLVESGGGSVQAGGSLRLSCAAS }} \frac{\text { CDR1 }}{\text { GDT--YGSYWMG WFRQAPGKEREGVA }}$ OVOLVESGGGLVOPGGSLRLSCAAS GDT---YGSYWMG WFROAPGOGLEAVA QVQLVESGGGLVQPGGSLRLSCAAS GGSEYSYSTFSLG WFRQAPGQGLEAVA

$\frac{\text { CDR2 }}{\text { AINRGGGYTVYADSVKG }} \frac{\text { FR3 }}{\text { RFTISRDTAKNTVYLQMNSLRPDDTADYYCAA }}$ AINRGGGYTVYADSVKG RFTISRDNSKNTLYLOMNSL RAEDTAVYYCAA AINRGGGYTVYADSVKG RFTISRDNSKNTLYLQMNSLRAEDTA VYYCAA
AIASMGGLTYYADSVKG RFTISRDNSKNTLYLQMNSLRAEDTAVYYCAA

$\frac{\text { CDR3 }}{\text { S-GVLGGLHEDW-FNY }} \frac{\text { FR4 }}{\text { WGQGTQVTVSS }} \frac{\text { His }_{6}}{\text { HHHHHH }}$ S-GVLGGLHEDW-FNY WGQGTLVTVSS HНHHHH VRGYFMRLPSSHNFRY WGQGTLVTVSS HHHHHH in PBS. The Nanobodies obtained after each purification step were visualized on Coomassie blue-stained gels (Supplemental Fig. 1; supplemental materials are available online only at http://jnm.snmjournals.org). The band for the Nanobodies was already visible in periplasmic extracts, and the use of the immobilized metal affinity chromatography and size-exclusion chromatography 2-step purification scheme yielded homogeneous preparations of the Nanobody with greater than $95 \%$ purity.

Affinities of the purified Nanobodies to immobilized CEA protein as measured by SPR are shown in Table 1. The NbCEA5 and the humanized CEA5 graft Nanobody bind to the CEA target protein with affinities in the nanomolar range, although an approximately 30-fold decrease in affinity is observed on grafting of the NbCEA5 antigen-binding loops onto the humanized scaffold. Binding of the humanized scaffold to the CEA target is not detectable by SPR under these conditions.

The binding of the 3 Nanobodies to immobilized CEA protein is also determined by ELISA (Fig. 2). Specific binding of NbCEA5 and humanized CEA5 graft to CEA protein was observed $(P<0.0001$ for both, as compared with background signal), although the signal was slightly lower for the grafted Nanobody. The humanized scaffold failed to recognize the CEA protein in ELISA $(P=0.1332$, as compared with background signal).

The capacity of the Nanobodies to associate with CEA expressed on live, nonfixed cells was evaluated by flow cytometry (Fig. 3). Cell lines with different CEA expression levels were chosen to assess the antigen specificity and selectivity of the Nanobodies. The humanized scaffold did not interact with any of the cells. The NbCEA5 and humanized CEA5 graft did not bind wild-type CHO cells but did recognize CEA-transfected $\mathrm{CHO}$ cells and CEApositive LS174T cells. In addition, the relative shifts of the fluorescence intensity peak indicate that the highest amount of binding occurred with NbCEA5 and that the humanized CEA5 graft has a slightly decreased binding capacity.

Additional competition studies demonstrated that a 10fold excess of NbCEA5 prevented the NbCEA5-Myc Nanobody from targeting CEA, as evidenced by ELISA on CEA protein (Supplemental Fig. 2A) and flow cytometry on CEA-expressing LS174T cells (Supplemental Fig. 2B). Under the same conditions, the humanized scaffold failed to compete with the NbCEA5-Myc for the CEA target in ELISA and flow cytometry (Supplemental Figs. $2 \mathrm{~A}$ and $2 \mathrm{C}$ ). However, in both tests, a strong competition between NbCEA5-Myc and the humanized CEA5 graft, leading to near-background background signals, was noticed (Supplemental Figs. 2A and 2D). This clearly indicates that NbCEA5 and the humanized CEA5 graft are highly specific for CEA, can compete for each other, and recognize the same epitope.

\section{Nanobody ${ }^{99 m}$ Tc Labeling and In Vitro Binding Characteristics}

The thermal stabilities of the 3 Nanobodies are shown in Table 1 and are in agreement with previous reports $(4,15)$. NbCEA5 and the humanized scaffold possess a melting temperature of $70^{\circ} \mathrm{C}$ or more. Significantly, the melting temperature is not affected after grafting of the NbCEA5 antigen-binding loops onto the humanized scaffold.

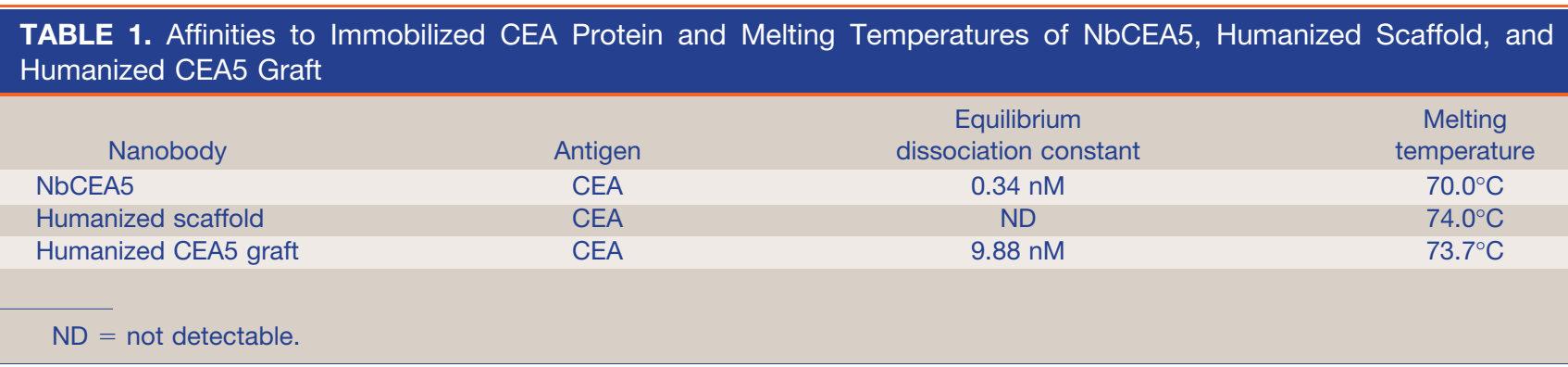




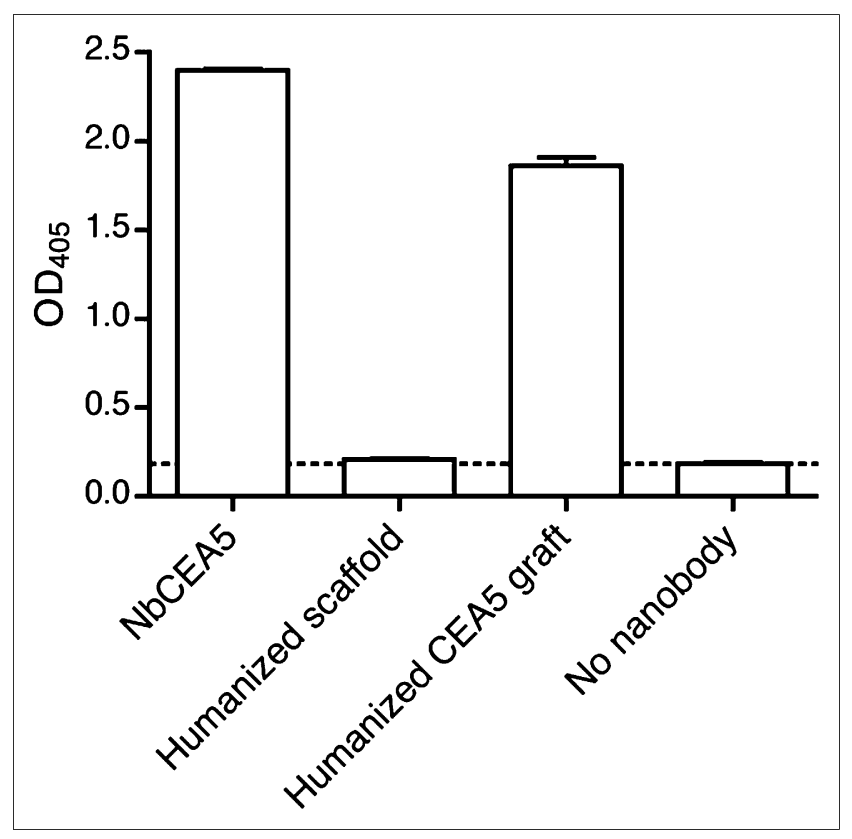

FIGURE 2. Specific binding of Nanobody NbCEA5 and humanized CEA5 graft, but not humanized scaffold, to immobilized CEA protein, as determined by ELISA. Data are presented as mean $\pm \mathrm{SD}(n=3)$. $\mathrm{OD}_{405}=$ optical density at $405 \mathrm{~nm}$.

Second, Nanobodies were labeled efficiently with ${ }^{99 \mathrm{~m}} \mathrm{Tc}$ using tricarbonyl chemistry. The radiochemical purity of 99mTc-labeled NbCEA5, humanized scaffold, and humanized CEA5 graft before purification was $95.81 \% \pm 0.69 \%$, $96.03 \% \pm 1.05 \%$, and $98.4 \% \pm 0.44 \%$, respectively. Unincorporated ${ }^{99} \mathrm{~m} \mathrm{Tc}(\mathrm{CO})_{3}$ was removed by gel filtration, after which radiochemical purity was greater than $99 \%$ for all 3 labeled Nanobodies, as determined by instant thinlayer chromatography.

The specificity of the purified ${ }^{99 \mathrm{~m}} \mathrm{Tc}-\mathrm{Nanobodies}$ for CEA was assessed by a binding assay to $\mathrm{CHO}$ (negative control), CEA-transfected CHO cells, or purified CEA protein. Results are shown in Figure 4 and correspond well to those obtained using ELISA and flow cytometry with unlabeled Nanobodies. ${ }^{99 \mathrm{~m}} \mathrm{Tc}-\mathrm{NbCEA} 5$ and ${ }^{99 \mathrm{~m}}$ Tc-humanized CEA5 grafts bind efficiently to both purified CEA protein and CEA-expressing $\mathrm{CHO}$ cells but fail to interact with CEA-negative $\mathrm{CHO}$ cells. Binding of ${ }^{99 \mathrm{~m}} \mathrm{Tc}$-humanized scaffold to CEA protein or CEA-expressing cells is negligible.

\section{Blood Clearance and Pinhole SPECT/Micro-CT Analysis of $99 \mathrm{~m}$ Tc-Nanobodies in Xenografted Mice}

All ${ }^{99 \mathrm{~m} T c-l a b e l e d}$ Nanobodies are cleared equally quickly from the blood, yielding similar biphasic blood curves (Supplemental Fig. 3). At $1 \mathrm{~h}$ after injection, \%IA/ total blood volume was less than or equal to 3 and not significantly different $(P>0.1)$ for the 3 Nanobodies.

Pinhole SPECT/micro-CT images of LS174T xenografted mice are depicted in Figure 5, and the results of image quantifications are summarized in Table 2. Images acquired at $1 \mathrm{~h}$ after injection showed intense uptake of all ${ }^{99 \mathrm{~m}} \mathrm{Tc}-$ Nanobodies in the kidney cortex and the bladder, as expected for small renal-filtered tracers. Signals are low and comparable in the liver, lungs, and muscle for all 3 compounds. Also, tracer elimination (excluding urinary activity in the bladder) from the body was similar for the 3 tracers: $21.13 \pm 11.22 \%$ IA for ${ }^{99 m}$ Tc-NbCEA5, $15.38 \pm$ $1.49 \%$ IA for ${ }^{99 \mathrm{~m} T c-l a b e l e d ~ h u m a n i z e d ~ s c a f f o l d, ~ a n d ~} 13.37 \pm$ $8.49 \%$ IA for ${ }^{99 \mathrm{~m} T c-l a b e l e d}$ humanized CEA5 graft $(P>$ 0.05 for all comparisons).
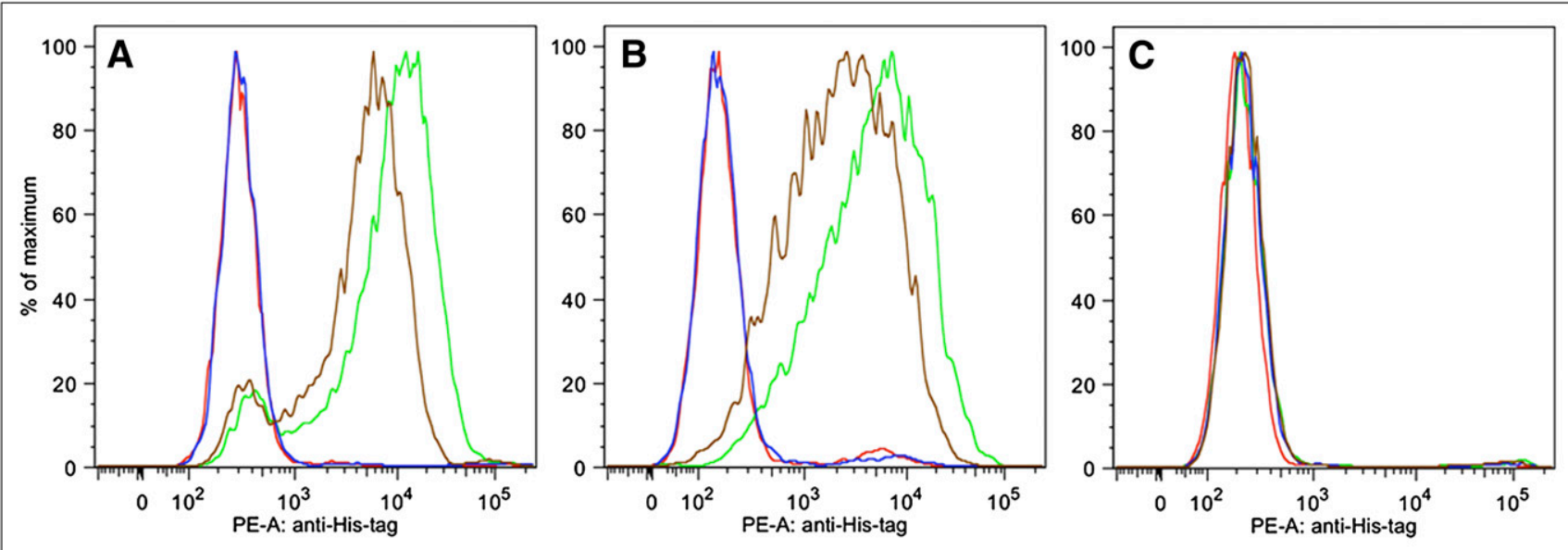

FIGURE 3. Binding of Nanobody NbCEA5 (green line) and humanized CEA5 graft (brown line), but not humanized scaffold (blue line), to CEA-transfected CHO (A) and CEA-expressing LS174T (B) cells, as determined by flow cytometry. No binding was observed to CEA-negative $\mathrm{CHO}$ cells $(\mathrm{C})$. Red lines in A-C represent background staining (no Nanobody added). PE-A = phycoerythrine-A. 
FIGURE 4. Specific binding of purified $99 \mathrm{~m}$ Tc-labeled Nanobody $99 \mathrm{~m}$ TcNbCEA5 and 99mTc-humanized CEA5 graft, but not ${ }^{99 m}$ Tc-humanized scaffold, to immobilized CEA protein $(A)$ and CEA-transfected cells (B). Binding to CEA-negative $\mathrm{CHO}$ cells was negligible for all ${ }^{99 m}$ Tc-labeled Nanobodies (C). Data are presented as mean $\pm \mathrm{SD}(n=6)$.

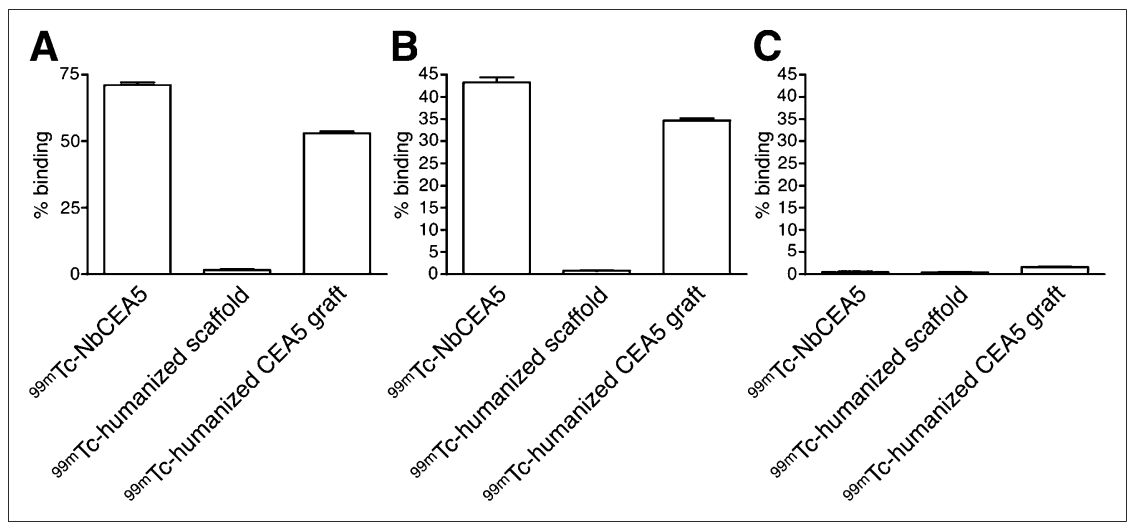

More importantly, intense tumor uptake, $7.09 \pm 1.36$ $\% \mathrm{IA} / \mathrm{cm}^{3}$ and $6.15 \pm 2.33 \% \mathrm{IA} / \mathrm{cm}^{3}$, respectively, was observed at $1 \mathrm{~h}$ after injection for both ${ }^{99 \mathrm{~m} T c-N b C E A 5}$ and ${ }^{99 \mathrm{~m}}$ Tc-labeled humanized CEA5 grafts, whereas there was marginal uptake for the ${ }^{99 \mathrm{~m}}$ Tc-labeled humanized scaffold in the CEA-positive LS174T tumor (Fig. 5). Interestingly, tumor uptake of ${ }^{99 \mathrm{~m} T c-l a b e l e d ~ N b C E A 5}$ in CEA-positive tumors was not significantly different from that of ${ }^{99 m}$ Tc-labeled humanized CEA5 grafts $(P=0.4014)$. In total, the efficient tumor targeting combined with low background signals due to rapid tracer clearance resulted in high tumor-to-muscle ratios for both the original (39.25 \pm
27.64) and the humanized CEA-targeting Nanobody tracer $(7.74 \pm 3.05)$.

\section{DISCUSSION}

Fast, sensitive, and reliable in vivo detection at an early stage of the disease remains a major challenge in cancer diagnosis. It seems that Nanobodies, with their unique biophysical and pharmacokinetic properties, are ideally suited to fulfill a prominent future role as a tool for the diagnosis of cancer. The combination of Nanobody uptake by tumors and rapid elimination from the circulation matches well with the half-life of radionuclides such as

FIGURE 5. Representative fused pinhole SPECT/micro-CT images $1 \mathrm{~h}$ after injection of intravenously injected 99mTc-labeled Nanobodies in xenografted mice show high uptake of both 99mTc-NbCEA5 and 99mTc-humanized CEA5 graft in CEA-positive LS174T tumors. Tumor uptake of ${ }^{99 m}$ Tc-humanized scaffold was low.

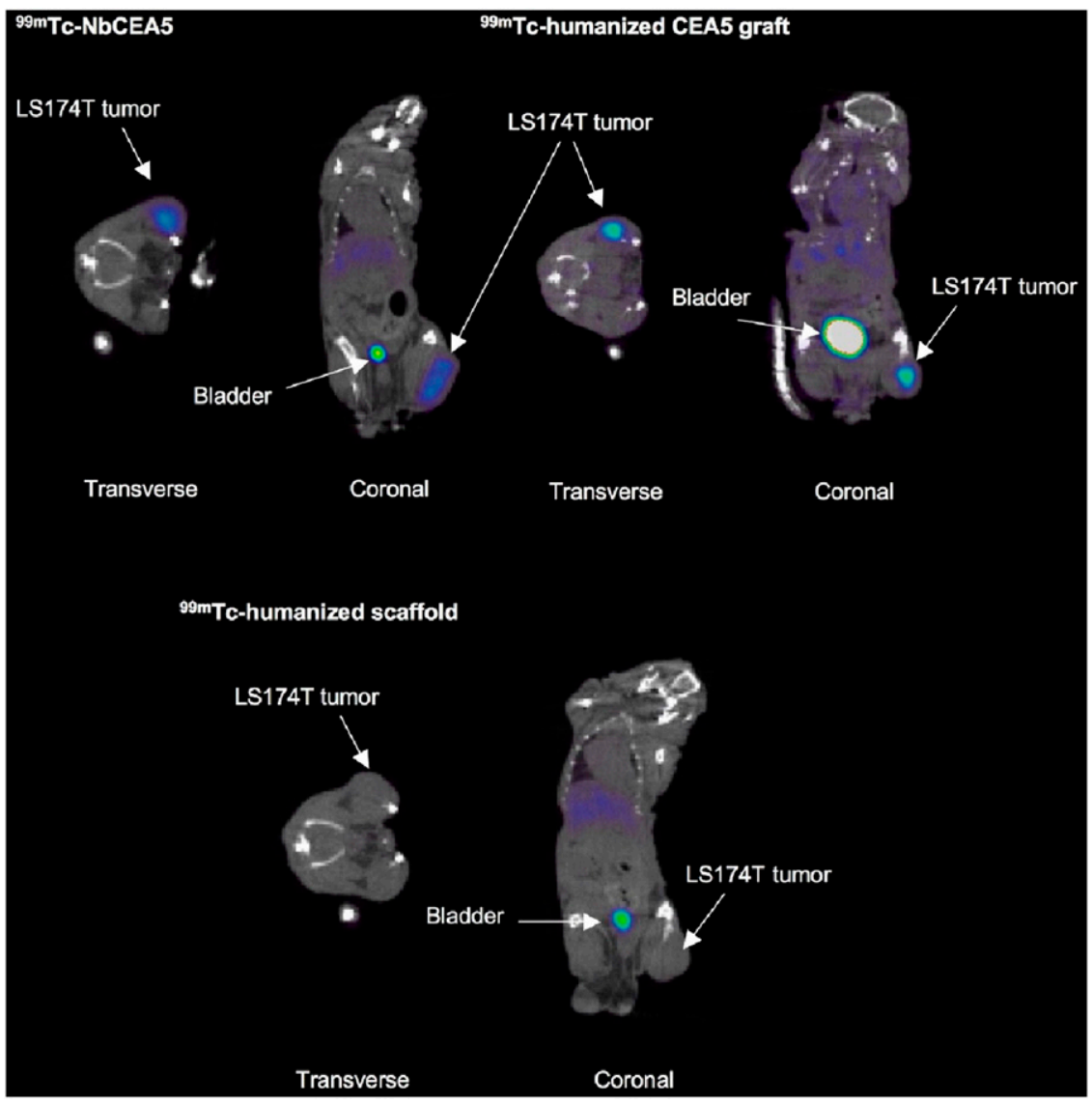


TABLE 2. Uptake Values of $99 \mathrm{mTc}-\mathrm{NbCEA5}$, ${ }^{99 \mathrm{mTC}}$ Humanized Scaffold, and ${ }^{99 m T c-H u m a n i z e d ~ C E A 5 ~}$ Graft Based on Pinhole SPECT/Micro-CT at

1 Hour After Injection

\begin{tabular}{|c|c|c|c|}
\hline Tissue & $\begin{array}{c}\text { 99mTc-NbCEA5 } \\
\left(\% \mathrm{IA} / \mathrm{cm}^{3}\right)^{\star}\end{array}$ & $\begin{array}{l}59 \mathrm{~m} \text { Tc-humanized } \\
\text { scaffold }\left(\% \mid \mathrm{A} / \mathrm{cm}^{3}\right)\end{array}$ & $\begin{array}{l}\text { 9mTc-humanized } \\
\text { CEA5 graft } \\
\left(\% \mathrm{I} / \mathrm{cm}^{3}\right)\end{array}$ \\
\hline Tumor & $7.09 \pm 1.36$ & $0.21 \pm 0.05$ & $6.15 \pm 2.33$ \\
\hline Muscle & $0.16 \pm 0.13$ & $0.26 \pm 0.03$ & $0.83 \pm 0.06$ \\
\hline Kidney & $88.01 \pm 12.16$ & $136.66 \pm 22.55$ & $87.05 \pm 6.85$ \\
\hline Lung & $1.49 \pm 0.50$ & $1.00 \pm 0.19$ & $2.19 \pm 1.25$ \\
\hline Liver & $2.19 \pm 0.67$ & $2.25 \pm 1.09$ & $3.09 \pm 0.49$ \\
\hline
\end{tabular}

${ }^{99 \mathrm{~m}}$ Tc. In addition, Nanobodies are in general robust and unusually stable at elevated temperature (15), and the crystal structures of the Nanobodies demonstrate that the $\mathrm{His}_{6}$ tail at their $\mathrm{C}$ terminus is located on the opposite side of the antigen-binding site (15). Previous experiments have indicated that Nanobodies site-specifically labeled with ${ }^{99 \mathrm{~m}} \mathrm{Tc}$ at this $\mathrm{His}_{6}$ tag using tricarbonyl chemistry (22) are stable and functional both in vitro and in vivo. ${ }^{99 \mathrm{~m}} \mathrm{Tc}-$ Nanobodies against CEA and epidermal growth factor receptor effectively targeted CEA and epidermal growth factor receptor-positive tumors, and unbound Nanobodies are rapidly cleared from the blood in mice (7-9). Knowing that the current clinical practice to characterize receptor status of cancer relies solely on invasive biopsy, the need for more specific, targeted imaging tracers in nuclear medicine is increasing and the Nanobody technology could offer a wide range of candidate new probes.

Because of their small size and the high degree of identity of their framework sequence to human $\mathrm{V}_{\mathrm{H}}$ proteins, it is expected that Nanobodies will exhibit a low immunogenicity (23). In addition, although no experimental data are available to determine whether nonhumanized Nanobodies are immunogenic in humans, intravenous injections of nonhumanized Nanobodies in mice at tracer doses did not stimulate antibody or cellular immune responses (24). Nevertheless, a translation to clinical applications will require maximal humanization of the Nanobody, preferably without compromising any of its affinity, specificity, and stability. Nanobodies and human $\mathrm{V}_{\mathrm{H}}$ domains differ in about 10 surface-exposed framework residues. Several of these substitutions involve a mutation from hydrophobic to more hydrophilic residues and assist in the solubility of Nanobodies (11). With the aim of approaching the structure of a human $\mathrm{V}_{\mathrm{H}}$, we recently proposed a strategy to manufacture a humanized Nanobody with maximal retention of stability and antigen-binding capacity (15). This approach is based on the grafting of the antigen-binding loops of a Nanobody, with antigen specificity of interest, onto a universal humanized Nanobody scaffold (h-
NbBcII10 $10_{\text {FGLA }}$ ). This universal scaffold accepts such loop grafts without losing (thermal) stability, functionality, and antigen specificity. In addition, we also demonstrated in this study that this humanized scaffold has a favorable biodistribution in vivo, with fast clearance and low retention in all organs except the kidneys.

As a proof of principle, we grafted successfully the antigen-binding loops of a CEA-targeting Nanobody onto our humanized Nanobody scaffold. An extensive in vitro characterization (SPR, ELISA, and flow cytometry measurements of CEA targeted by Nanobodies directly or in competition studies) showed that the new humanized CEA5 graft Nanobody maintains a high affinity and specificity for CEA, although some loss of CEA-binding strength was inevitable. All Nanobodies were heat-stable and could be efficiently labeled with ${ }^{99 \mathrm{~m}} \mathrm{Tc}$, with full retention of their functionality (i.e., specific recognition of CEA antigen). We have already emphasized the possible reduction of antigen affinity after grafting because framework residues might also participate directly in antigen recognition or indirectly by proper positioning of the antigen-binding loops in the Nanobody (15). In vivo, however, both ${ }^{99 m}$ Tc-NbCEA5 and 99mTc-humanized CEA graft showed good targeting of a CEA-positive tumor, with a tumor uptake of $6.15 \% \mathrm{IA} /$ $\mathrm{cm}^{3}$ for the humanized CEA5 grafts. Tumor uptake of antibodies or antibody fragments after intravenous delivery is a complex combination of various biologic factors of which the affinity for the antigen is only 1 aspect, as discussed by Rudnick et al. (25): factors such as capillary extravasation, tumor penetration, tracer internalization and metabolism, tumor interstitial pressure, abundance, and shedding of targeted receptors can be equally important in determining tumor-targeting levels. Good tumor-tobackground ratios were already obtained at $1 \mathrm{~h}$ after injection for both CEA-targeting Nanobodies, as explained by the rapid elimination of unbound tracer from the circulation. ${ }^{99 \mathrm{~m}} \mathrm{Tc}$-labeled Nanobodies are rapidly cleared from the blood, mainly via the kidneys. This property is typical of peptides and small proteins whose molecular weight is below the threshold that can be filtered by the glomerular membrane $(<60 \mathrm{kDa})$. So, although NbCEA5 CDR grafting results in a partial loss of affinity (albeit still in the nanomolar range), the in vitro and in vivo CEAtargeting potential is retained.

\section{CONCLUSION}

Nanobodies can be successfully humanized by loopgrafting. These humanized, grafted Nanobodies are functional both in vitro and in vivo and can be used as tracers for imaging purposes with reduced risks for immunogenicity. A small loss in antigen-binding strength was observed in all in vitro studies; however, in vivo tumor targeting was hardly affected.

Therefore, it seems that, in the future, the antigenbinding loops of a broad variety of Nanobodies against 
different targets can be considered for grafting onto this universal, humanized scaffold. This approach represents a generic tool that allows complete standardizationincluding standardization of radiolabeling-because uniform chemical modifications and labeling protocols could be optimized for this particular backbone structure. We anticipate that this strategy can lead to a fast translation of Nanobodies to clinical imaging.

\section{ACKNOWLEDGMENTS}

CHO-CEA cells were kindly provided by Drs. Motomu Kuroki and Masahide Kuroki, Fukuoka University, Japan. This work is supported in part by an IWT grant. The research at ICMI was funded by the Interuniversity Attraction Poles Program, Belgian State, Belgian Science Policy.

\section{REFERENCES}

1. Hamers-Casterman C, Atarhouch T, Muyldermans S, et al. Naturally occurring antibodies devoid of light chains. Nature. 1993;363:446-448.

2. Baral TN, Magez S, Stijlemans B, et al. Experimental therapy of African trypanosomiasis with a nanobody-conjugated human trypanolytic factor. Nat Med. 2006;12:580-584.

3. Revets H, De Baetselier P, Muyldermans S. Nanobodies as novel agents for cancer therapy. Expert Opin Biol Ther. 2005;5:111-124.

4. Cortez-Retamozo V, Backmann N, Senter PD, et al. Efficient cancer therapy with a nanobody-based conjugate. Cancer Res. 2004;64:2853-2857.

5. Rothbauer U, Zolghadr K, Tillib S, et al. Targeting and tracing antigens in live cells with fluorescent nanobodies. Nat Methods. 2006;3:887-889.

6. De Groeve K, Deschacht N, De Koninck C, et al. Nanobodies as tools for in vivo imaging of specific immune cell types. J Nucl Med. 2010;51:782-789.

7. Cortez-Retamozo V, Lahoutte T, Caveliers V, et al. ${ }^{99 \mathrm{~m}} \mathrm{Tc}$-labeled Nanobodies: a new type of targeted probes for imaging antigen expression. Curr Radiopharmaceuticals. 2008;1:37-41.

8. Gainkam LO, Huang L, Caveliers V, et al. Comparison of the biodistribution and tumor targeting of two ${ }^{99 \mathrm{~m}} \mathrm{Tc}$-labeled anti-EGFR nanobodies in mice, using pinhole SPECT/micro-CT. J Nucl Med. 2008;49:788-795.

9. Huang L, Gainkam LO, Caveliers V, et al. SPECT imaging with ${ }^{99 \mathrm{~m}}$ Tc-labeled EGFR-specific nanobody for in vivo monitoring of EGFR expression. Mol Imaging Biol. 2008;10:167-175.
10. De Groot AS, Scott DW. Immunogenicity of protein therapeutics. Trends Immunol. 2007;28:482-490.

11. Muyldermans S, Atarhouch T, Saldanha J, Barbosa JA, Hamers R. Sequence and structure of $\mathrm{V}_{\mathrm{H}}$ domain from naturally occurring camel heavy chain immunoglobulins lacking light chains. Protein Eng. 1994;7:1129-1135.

12. Davies J, Riechmann L. 'Camelising' human antibody fragments: NMR studies on $\mathrm{V}_{\mathrm{H}}$ domains. FEBS Lett. 1994;339:285-290.

13. Conrath K, Vincke C, Stijlemans B, et al. Antigen binding and solubility effects upon the veneering of a camel VHH in framework-2 to mimic a VH. J Mol Biol. 2005;350:112-125.

14. Desmyter A, Decanniere K, Muyldermans S, Wyns L. Antigen specificity and high affinity binding provided by one single loop of a camel single-domain antibody. J Biol Chem. 2001;276:26285-26290.

15. Vincke C, Loris R, Saerens D, Martinez-Rodriguez S, Muyldermans S, Conrath $\mathrm{K}$. General strategy to humanize a camelid single-domain antibody and identification of a universal humanized nanobody scaffold. J Biol Chem. 2009;284: 3273-3284.

16. Duffy MJ. Carcinoembryonic antigen as a marker for colorectal cancer: is it clinically useful? Clin Chem. 2001;47:624-630.

17. Saerens D, Pellis M, Loris R, et al. Identification of a universal VHH framework to graft non-canonical antigen-binding loops of camel single-domain antibodies. J Mol Biol. 2005;352:597-607.

18. Conrath KE, Lauwereys M, Galleni M, et al. $\beta$-lactamase inhibitors derived from single-domain antibody fragments elicited in the camelidae. Antimicrob Agents Chemother. 2001;45:2807-2812.

19. Oikawa S, Inuzuka C, Kuroki M, Matsuoka Y, Kosaki G, Nakazato H. Cell adhesion activity of non-specific cross-reacting antigen (NCA) and carcinoembryonic antigen (CEA) expressed on $\mathrm{CHO}$ cell surface: homophilic and heterophilic adhesion. Biochem Biophys Res Commun. 1989;164:39-45.

20. Vanhove C, Defrise M, Bossuyt A, Lahoutte T. Improved quantification in single-pinhole and multiple-pinhole SPECT using micro-CT information. Eur $J$ Nucl Med Mol Imaging. 2009;36:1049-1063.

21. Loening AM, Gambhir SS. AMIDE: a free software tool for multimodality medical image analysis. Mol Imaging. 2003;2:131-137.

22. Waibel R, Alberto R, Willuda J, et al. Stable one-step technetium- $99 \mathrm{~m}$ labeling of His-tagged recombinant proteins with a novel Tc(I)-carbonyl complex. Nat Biotechnol. 1999;17:897-901.

23. Harmsen MM, De Haard HJ. Properties, production, and applications of camelid single-domain antibody fragments. Appl Microbiol Biotechnol. 2007;77:13-22.

24. Cortez-Retamozo V, Lauwereys M, Hassanzadeh Gh G, et al. Efficient tumor targeting by single-domain antibody fragments of camels. Int J Cancer. 2002;98: $456-462$.

25. Rudnick SI, Adams GP. Affinity and avidity in antibody-based tumor targeting. Cancer Biother Radiopharm. 2009;24:155-161. 